\title{
Numerical Study of Mixed Convection of the Nanofluids in Two-Sided Lid-Driven Square Cavity with a Pair of Triangular Heating Cylinders
}

\author{
Zoubair Boulahia, Abderrahim Wakif, and Rachid Sehaqui \\ Faculty of Sciences Aïn Chock, Laboratory of Mechanics, Hassan II University, BP 5366, Maarif, 20100 Casablanca, Morocco \\ Correspondence should be addressed to Zoubair Boulahia; boulahia.zoubair@gmail.com
}

Received 29 November 2015; Accepted 14 January 2016

Academic Editor: Junling $\mathrm{Hu}$

Copyright (C) 2016 Zoubair Boulahia et al. This is an open access article distributed under the Creative Commons Attribution License, which permits unrestricted use, distribution, and reproduction in any medium, provided the original work is properly cited.

A numerical study is carried out concerning mixed convection of the nanofluid in two-sided lid-driven square cavity with a pair of triangular heat sources. The upper and bottom moving walls are thermally insulated while the left and right walls are cooled at constant temperature. Two-dimensional Navier-Stokes and energy equations are solved using the finite volume discretization method with SIMPLE algorithm. The method used is validated against previous works. Two cases were considered depending on the direction of moving walls. Effects of various design parameters such as Richardson number $(0.1 \leq \mathrm{Ri} \leq 100)$, nanoparticle volume fraction $(0 \leq \varphi \leq 0.05)$, and size $\left(25 \mathrm{~nm} \leq d_{p} \leq 145 \mathrm{~nm}\right)$ and type $\left(\mathrm{Cu}, \mathrm{Al}_{2} \mathrm{O}_{3}, \mathrm{TiO}_{2}\right)$ of nanoparticles on the heat transfer rate are investigated. The results of this investigation illustrate that, by reducing the diameter of the nanoparticles and $\mathrm{Ri}$, the heat transfer rate increases. Moreover, it is found that by changing horizontal direction of the moving walls the heat transfer rate variation is negligible.

\section{Introduction}

Mixed convection problem with lid-driven flows in enclosures occurs in many engineering applications such as heating and cooling nuclear systems of reactors, lubrication technologies, cooling of electronic devices, and heat exchangers. There are many studies concerning the heat transfer in rectangular or square cavities driven by buoyancy and shear which have been studied extensively in the literature. Work of Oztop et al. [1] can be mentioned as example of such studies, in which they numerically studied mixed convection in square cavities with two moving walls. Their results suggest that when the vertical walls move upwards in the same direction, the heat transfer decreases significantly compared to when the vertical walls move in opposite directions. Work of Khanafer and Aithal [2] was concentrated on the effect of mixed convection flow and heat transfer characteristics in a lid-driven cavity with a circular body inside. The results showed that the average Nusselt number increases with an increase in the radius of the cylinder for various Richardson numbers and the optimal heat transfer results are obtained when placing the cylinder near the bottom wall. Islam et al. [3] investigated a numerical study on a lid-driven cavity with a heated square blockage. They found that size, location, and Richardson number of the heater eccentricities affect the average Nusselt number of heater. Kalteh et al. [4] considered laminar mixed convection of nanofluid in a lid-driven square cavity with a triangular heat source. Their simulations indicate that increasing the volume fraction, the nanoparticles diameter, and Reynolds number leads to an increase in average Nusselt number.

El Abdallaoui et al. [5] performed a numerical simulation of natural convection between a decentered triangular heating cylinder and centered triangular heating cylinder [6] in a square outer cylinder filled with a pure fluid or a nanofluid using the lattice Boltzmann method. The results indicate that the horizontal displacement from the centered position to decentered position leads to a considerable increase of heat transfer at weak Rayleigh and the vertical displacement has most important effect on heat transfer at high values of Rayleigh. Oztop et al. [7] investigated mixed convection in a lid-driven cavity with a cylindrical blockage inside. 
TABLE 1: Thermophysical properties of water and nanoparticles at $T=300 \mathrm{~K}$ [14].

\begin{tabular}{lcccccc}
\hline & $C_{p}(\mathrm{~J} / \mathrm{KgK})$ & $\rho\left(\mathrm{Kg} / \mathrm{m}^{3}\right)$ & $K(\mathrm{~W} / \mathrm{mK})$ & $\beta \times 10^{5}\left(\mathrm{~K}^{-1}\right)$ & $\mu \times 10^{6}\left(\mathrm{kgm}^{-1} \mathrm{~s}^{-1}\right)$ & $d_{p}\left(\mathrm{~nm}^{2}\right)$ \\
\hline Copper $(\mathrm{Cu})$ & 385 & 8933 & 401 & 1.67 & - & - \\
$\mathrm{Al}_{2} \mathrm{O}_{3}$ & 765 & 3970 & 36 & 0.85 & - & $25,85,145$ \\
$\mathrm{TiO}_{2}$ & 710 & 4157 & 8.4 & 0.9 & 25 \\
Water $\left(\mathrm{H}_{2} \mathrm{O}\right)$ & 4179 & 997.1 & 0.613 & 27.6 & 855 \\
\hline
\end{tabular}

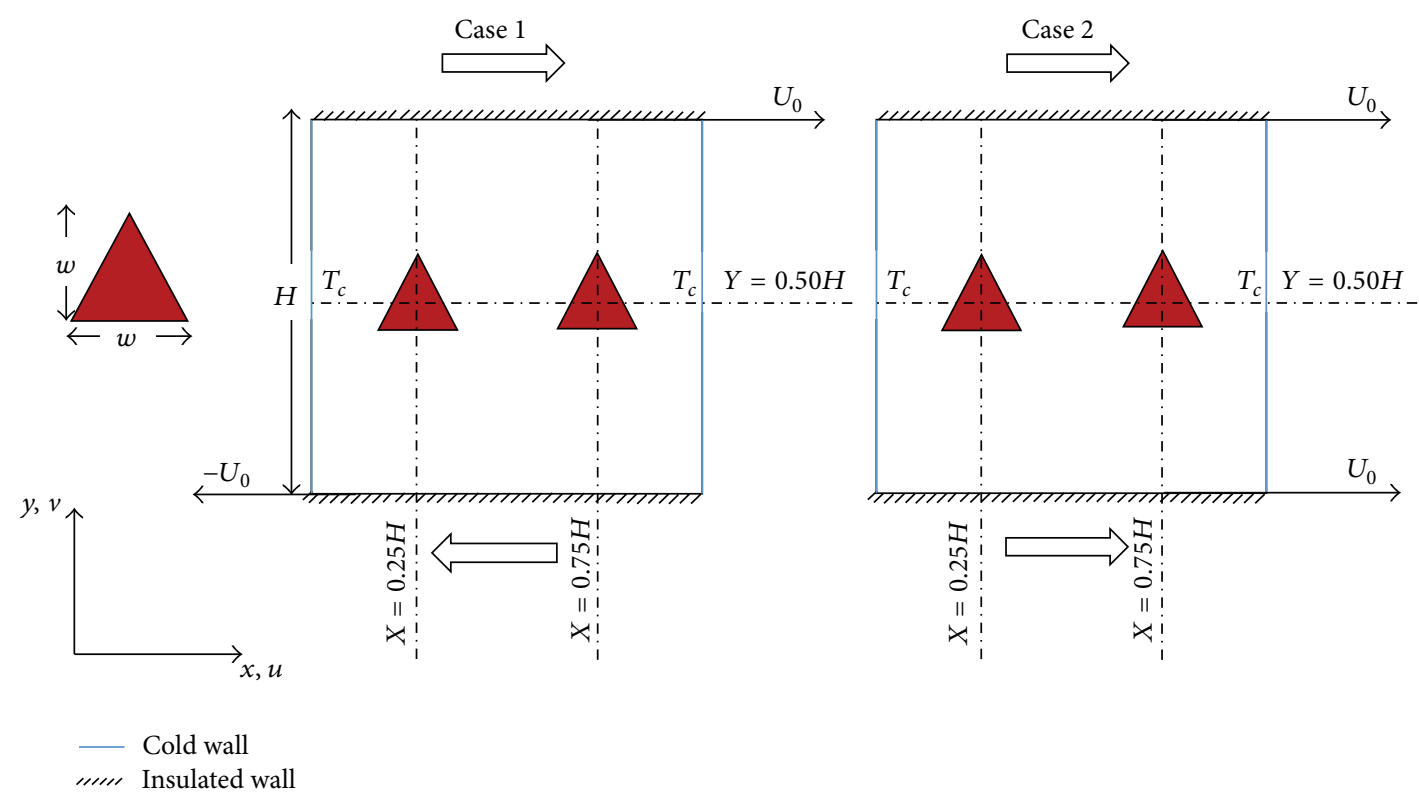

FIGURE 1: Schematic of the cavity with a pair of triangular heat sources and boundary conditions.

The vertical left sidewall was moving up or down. The computation was carried out for wide ranges of Richardson number, inner cylinder diameter, and center and location of the inner cylinder. They concluded that the most effective parameter on flow field and temperature distribution was the direction of the moving lid.

In the present study, the main aim is to examine the mixed convection heat transfer of nanofluid in a square enclosure with a pair of triangular heat sources. The first case under investigation is characterized by the numerical models used in our study. The computational procedure elaborated in this study is validated against the numerical results of other investigations. We studied the effects of various design parameters such as Richardson number $(0.1 \leq \mathrm{Ri} \leq 100)$, nanoparticle volume fraction $(0 \leq \varphi \leq 0.05)$, and size $\left(25 \mathrm{~nm} \leq d_{p} \leq 145 \mathrm{~nm}\right)$ and type $\left(\mathrm{Cu}, \mathrm{Al}_{2} \mathrm{O}_{3}, \mathrm{TiO}_{2}\right)$ of nanoparticles on the heat transfer. The new models of the thermal conductivity and effective viscosity investigated by Corcione [8] are used to estimate thermophysical proprieties of the nanofluid. Our numerical results are presented in the form of plots of isotherms, streamlines, and average Nusselt numbers to show the influence of nanofluid and design parameters.

\section{Problem Statement}

The studied configurations and coordinate system of the considered enclosure in the present study are shown in
Figure 1. It is a two-sided lid-driven square cavity with a pair of triangular heating cylinders, filled with nanofluids. The triangular heat source is maintained at a temperature $T_{h}=$ $310 \mathrm{~K}$. The vertical walls are cooled at constant temperatures $T_{c}=290 \mathrm{~K}$. Two different cases were considered as shown in Figure 1. In Case 1, the upper wall (adiabatic) is moving to the right while bottom wall (adiabatic) is moving to the left. In Case 2, the direction of the two moving walls is the same. It is assumed that the nanofluid is Newtonian, incompressible, and laminar and the base fluid and the nanoparticles are in a thermal equilibrium state. The thermophysical properties of the nanofluid used in this study are evaluated at the average fluid temperature $\left(T_{c}+T_{h}\right) / 2$ as listed in Table 1 .

\section{Mathematical Formulation}

The governing equations including the two-dimensional transient equations of the continuity, momentum, and energy for an incompressible flow are expressed in the following format:

$$
\begin{gathered}
\frac{\partial u}{\partial x}+\frac{\partial v}{\partial y}=0 \\
\frac{\partial u}{\partial t}+u \frac{\partial u}{\partial x}+v \frac{\partial u}{\partial y}=-\frac{1}{\rho_{\mathrm{nf}}} \frac{\partial p}{\partial x}+\frac{\mu_{\mathrm{nf}}}{\rho_{\mathrm{nf}}}\left(\frac{\partial^{2} u}{\partial x^{2}}+\frac{\partial^{2} u}{\partial y^{2}}\right)
\end{gathered}
$$




$$
\begin{aligned}
\frac{\partial v}{\partial t}+u \frac{\partial v}{\partial x}+v \frac{\partial v}{\partial y}= & -\frac{1}{\rho_{\mathrm{nf}}} \frac{\partial p}{\partial y}+\frac{\mu_{\mathrm{nf}}}{\rho_{\mathrm{nf}}}\left(\frac{\partial^{2} v}{\partial x^{2}}+\frac{\partial^{2} v}{\partial y^{2}}\right) \\
& +\frac{(\rho \beta)_{\mathrm{nf}}}{\rho_{\mathrm{nf}}}\left(T-T_{c}\right), \\
\frac{\partial T}{\partial t}+u \frac{\partial T}{\partial x}+v \frac{\partial T}{\partial y}= & \alpha_{\mathrm{nf}}\left(\frac{\partial^{2} T}{\partial x^{2}}+\frac{\partial^{2} T}{\partial y^{2}}\right),
\end{aligned}
$$

where the nanofluid effective density, heat capacity, thermal expansion coefficient, and thermal diffusivity are calculated from the following equations $[9,10]$ :

$$
\begin{aligned}
\rho_{\mathrm{nf}} & =(1-\varphi) \rho_{f}+\varphi \rho_{s}, \\
\left(\rho C_{p}\right)_{\mathrm{nf}} & =(1-\varphi)\left(\rho C_{p}\right)_{f}+\varphi\left(\rho C_{p}\right)_{s}, \\
(\rho \beta)_{\mathrm{nf}} & =(1-\varphi)(\rho \beta)_{f}+\varphi(\rho \beta)_{s}, \\
\alpha_{\mathrm{nf}} & =\frac{k_{\mathrm{nf}}}{\left(\rho C_{p}\right)_{\mathrm{nf}}} .
\end{aligned}
$$

Corcione model [8-10] for dynamic viscosity and thermal conductivity of the nanofluid is given by

$$
\begin{aligned}
\mu_{\mathrm{nf}} & =\frac{\mu_{f}}{\left(1-34.87\left(d_{p} / d_{f}\right)^{-0.3} \varphi^{1.03}\right)}, \\
\frac{k_{\mathrm{nf}}}{k_{f}} & =1+4.4 \operatorname{Re}_{B}^{0.4} \operatorname{Pr}^{0.66}\left(\frac{T}{T_{f r}}\right)^{10}\left(\frac{k_{p}}{k_{f}}\right)^{0.03} \varphi^{0.66}, \\
\operatorname{Re}_{B} & =\frac{\rho_{f} u_{B} d_{p}}{\mu_{f}}, \\
u_{B} & =\frac{2 k_{b} T}{\pi \mu_{f} d_{p}^{2}} .
\end{aligned}
$$

All terms are defined in Nomenclature.

The boundary conditions for mixed convection are written as

$$
\begin{aligned}
u & =-U_{0} \text { or } U_{0}, \\
v & =0, \\
\frac{\partial T}{\partial y} & =0, \\
u & =U_{0}, \\
v & =0, \\
\frac{\partial T}{\partial y} & =0,
\end{aligned}
$$$$
\text { on bottom wall of the cavity, }
$$

on upper wall of the cavity,

$$
\begin{aligned}
& v=0, \\
& v=0, \\
& T=T_{c}, \\
& u=0, \\
& v=0, \\
& T=T_{c},
\end{aligned}
$$

on right wall of the cavity,

on left wall of the cavity.

The following dimensionless variables for mixed convection are defined based on properties of pure fluid:

$$
\begin{aligned}
\tau & =\frac{t}{H / U_{\text {ref }}}, \\
X & =\frac{x}{H}, \\
Y & =\frac{y}{H}, \\
U & =\frac{u}{U_{\text {ref }}}, \\
V & =\frac{v}{U_{\text {ref }}}, \\
P & =\frac{p}{\rho_{\mathrm{nf}} U_{\mathrm{ref}}^{2}}, \\
\theta & =\frac{T-T_{c}}{T_{h}-T_{c}},
\end{aligned}
$$

where $U_{\text {ref }}$ is considered to be $U_{0}$ for mixed convection. Dimensionless numbers for the system are defined as

$$
\begin{aligned}
& \mathrm{Re}=\frac{u_{\mathrm{ref}} H}{v_{\mathrm{nf}}}, \\
& \mathrm{Ri}=\frac{\mathrm{Gr}}{\mathrm{Re}^{2}}, \\
& \mathrm{Gr}=\frac{g \beta_{f}\left(T_{h}-T_{c}\right) H^{3}}{v_{f}^{2}}, \\
& \operatorname{Ra}=\mathrm{Gr} \cdot \operatorname{Pr}=\frac{g \beta_{f}\left(T_{h}-T_{c}\right) H^{3}}{\alpha_{f} v_{f}}, \\
& \operatorname{Pr}=\frac{v_{f}}{\alpha_{f}} .
\end{aligned}
$$


The governing equations (1) are written in the following dimensionless form:

$$
\begin{aligned}
& \frac{\partial U}{\partial X}+\frac{\partial V}{\partial Y}=0, \\
& \frac{\partial U}{\partial \tau}+U \frac{\partial U}{\partial X}+V \frac{\partial U}{\partial Y}=-\frac{\partial P}{\partial X} \\
& +\frac{1}{\operatorname{Re}} \frac{\rho_{f}}{\rho_{\mathrm{nf}}} \frac{\mu_{\mathrm{nf}}}{\mu_{f}}\left(\frac{\partial^{2} U}{\partial X^{2}}+\frac{\partial^{2} U}{\partial Y^{2}}\right), \\
& \frac{\partial V}{\partial \tau}+U \frac{\partial V}{\partial X}+V \frac{\partial V}{\partial Y}=-\frac{\partial p}{\partial Y} \\
& +\frac{1}{\operatorname{Re}} \frac{\rho_{f}}{\rho_{\mathrm{nf}}} \frac{\mu_{\mathrm{nf}}}{\mu_{f}}\left(\frac{\partial^{2} V}{\partial X^{2}}+\frac{\partial^{2} V}{\partial Y^{2}}\right) \\
& +\operatorname{Ri} \frac{(\rho \beta)_{\mathrm{nf}}}{\rho_{\mathrm{nf}} \beta_{f}} \theta \text {, } \\
& \frac{\partial \theta}{\partial \tau}+U \frac{\partial \theta}{\partial X}+V \frac{\partial \theta}{\partial Y}=\frac{1}{\operatorname{Re} \cdot \operatorname{Pr}} \frac{\alpha_{\mathrm{nf}}}{\alpha_{f}}\left(\frac{\partial^{2} \theta}{\partial X^{2}}+\frac{\partial^{2} \theta}{\partial Y^{2}}\right) .
\end{aligned}
$$

Dimensionless form of the boundary conditions can be written as

$$
\begin{aligned}
U & =1 \text { or }-1, \\
V & =0, \\
\frac{\partial \theta}{\partial y} & =0,
\end{aligned}
$$

on bottom wall of the cavity,

$$
\begin{gathered}
U=1, \\
V=0, \\
\frac{\partial \theta}{\partial y}=0,
\end{gathered}
$$$$
\text { on upper wall of the cavity, }
$$

$$
\begin{aligned}
& V=0, \\
& V=0, \\
& \theta=0,
\end{aligned}
$$

on right wall of the cavity,

$$
\begin{aligned}
& U=0, \\
& V=0, \\
& \theta=0,
\end{aligned}
$$

on left wall of the cavity.

The total mean Nusselt number of all cavity's wall is defined as

$$
\overline{\mathrm{Nu}}_{\mathrm{tot}}=\frac{1}{H} \int_{0}^{H} \frac{k_{\mathrm{nf}}(\varphi)}{k}\left\{\left|\frac{\partial \theta}{\partial X}\right|_{\text {left }}+\left|\frac{\partial \theta}{\partial X}\right|_{\text {right }}\right\} d Y .
$$

TABLE 2: Effect of the grid size on $\overline{\mathrm{Nu}}_{\text {tot }}$ for the cavity filled with the $\mathrm{Cu}$-water nanofluid $(\varphi=0.05)$ having a pair of triangular heat sources with size $W=w / H=0.2$ for case 1 (see Figure 1 ).

\begin{tabular}{lcccc}
\hline $\mathrm{Ri}$ & $63 \times 63$ & $83 \times 83$ & $103 \times 103$ & $123 \times 123$ \\
\hline 0.1 & 19.781 & 20.295 & 20.351 & 20.357 \\
100 & 8.921 & 9.377 & 9.438 & 9.439 \\
\hline
\end{tabular}

TABLE 3: Comparison of $\overline{\mathrm{Nu}}$ at the hot lid between the present results and those reported in the literature.

\begin{tabular}{lccc}
\hline \multirow{2}{*}{ Ri } & \multicolumn{3}{c}{ Average Nusselt number at the hot lid } \\
& Iwatsu et al. [13] & Oztop et al. [7] & Present \\
\hline 1.0 & 1.34 & 1.30 & $\mathbf{1 . 3 6}$ \\
0.0625 & 3.62 & 3.63 & $\mathbf{3 . 6 8}$ \\
0.01 & 6.29 & 6.34 & $\mathbf{6 . 2 9}$ \\
\hline
\end{tabular}

\section{Numerical Details}

The discretization procedure of the governing equations (7) and boundary conditions described by (8) is based on a finite volume formulation, given by Patankar [11] on a staggered grid. SIMPLE (Semi-Implicit Method for Pressure Linked Equations) is used to solve the coupled pressurevelocity equation while Hybrid Differencing Scheme (HDS) of Spalding [12] is used for the convective terms. Line by line application of TDMA (Tridiagonal Matrix Algorithm) method [12] is applied on equation systems until sum of the residuals became less than $10^{-6}$. The developed algorithm was implemented in FORTRAN program.

4.1. Grid Independence Study. In order to determine a proper grid for the numerical simulation, a square cavity filled with $\mathrm{Cu}$-water nanofluid $(\varphi=0.05)$ having a pair of triangular heat sources with size $w=0.2$ is analyzed in two extreme Richardson numbers $(\mathrm{Ra}=0.1$ and 100). The mean Nusselt number obtained using different grid numbers for particular cases is presented in Table 2. As can be observed from the table, a uniform $103 \times 103$ grid is sufficiently fine for the numerical calculation.

4.2. Validations. The present numerical scheme was validated against various numerical results available in the literature, two different heat convection problems are chosen. The first case is the numerical results of Iwatsu et al. [13] and Oztop et al. [7] for a top heated moving lid and bottom cooled square cavity filled with air $(\operatorname{Pr}=0.71)$. A $100 \times$ 100 mesh was used and the computations were done for three different Richardson numbers. Table 3 demonstrates an excellent comparison of the average Nusselt number between the present results and the numerical results found in the literature [7-13] with a maximum discrepancy of about $1.6 \%$. The second case is a mixed convection flow and heat transfer characteristics in a lid-driven cavity with a square heater inside. Figure 2 illustrates a comparison of the isotherms and streamlines between the present results and the results 

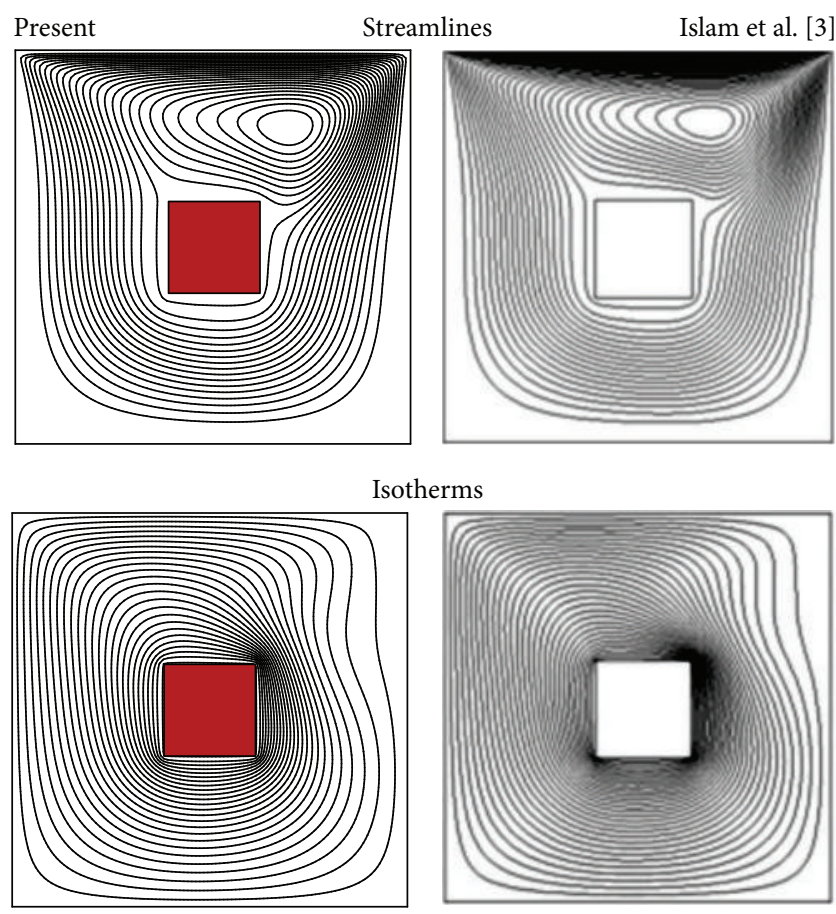

Figure 2: Comparison of the streamlines and isotherms between the present results and that of Islam et al. [3] $(h / H=0.25, \mathrm{Re}=$ 100 , and $\mathrm{Ri}=0.1$ ).

reported by Islam et al. [3] at Reynolds number $(\mathrm{Re}=100)$ and at Richardson numbers $(\mathrm{Ri}=0.1)$.

\section{Results and Discussion}

In this section, the numerical results for mixed convection heat transfer of the nanofluid in a square cavity with horizontal moving lids are discussed. Results pertinent to the effects of the moving wall direction of motion, volume fraction $(0 \leq$ $\varphi \leq 0.05)$, diameter $\left(25 \mathrm{~nm} \leq d_{p} \leq 145 \mathrm{~nm}\right)$, and type $\left(\mathrm{Cu}, \mathrm{Al}_{2} \mathrm{O}_{3}\right.$, and $\left.\mathrm{TiO}_{2}\right)$ of the nanoparticles on the fluid flow and heat transfer rate are presented for the square cavity with a pair of triangular heat sources placed horizontally (see Figure 1, Cases 1 and 2). Size of triangular heat sources is fixed at $w=0.2 \mathrm{H}$.

Figures 3(a) and 3(b) show the effects of the moving wall direction of motion on the streamlines and isotherms for various values of $\mathrm{Ri}$ ranging from 0.1 to 100 while Grashof number is fixed at $\mathrm{Gr}=10^{4}$. The cavity is filled with $\mathrm{Cu}$ water $(\varphi=5 \%)$, and for comparisons the streamlines and the isotherms for pure fluid and nanofluid are shown by dashed line and solid line, respectively. As shown in Figure 3, some differences are observed in streamlines and isotherms of pure fluid and nanofluid. We can explain this by the higher viscosity of nanofluid compared to that of the pure fluid which increases the diffusion of momentum in the nanofluid. Figure 3(a) shows that, at $\mathrm{Ri}=0.1$ for Case 1 , the flow field is characterized by a clockwise vortex created in the upper half of the enclosure, and another created in the lower half. These two vortexes are mostly generated by horizontal moving lids.
At this Ri, we can see in Figure 3(b) that the concentration of the isotherms, close to the pair of triangular heat sources, increases, which indicates that the heat transfer is through forced convection. By increasing $\mathrm{Ri}$, the effects of natural convection and buoyancy force become stronger leading to formation of a counterclockwise rotating eddy close to the left wall of the cavity. The effects of moving wall decrease by increasing $\mathrm{Ri}$. The uniformly distributed isotherms at high $\mathrm{Ri}$ show that the main heat transfer mechanism is through the natural convection.

Figure 3(a) shows that, at low Ri for Case 2, the flow field is characterized also by a clockwise vortex created in the upper half of the enclosure, and a counterclockwise vortex created in the lower half. These two vortices move to the right side due to an increase in lids speed. Figure 3(b) shows that the concentration of the isotherms, close to the pair of triangular heat sources and left wall of the cavity, increases. The isotherms move to the left side and the diffusion of heat to the right side decreases with increasing the speed of the horizontal lids (decreasing Ri). This observation shows the higher forced convection effects in the right side of the cavity. When Ri increases to 100, the effects of moving wall decrease due to the enhancement in the buoyancy force. A secondary eddy appears on the left indicates that the natural convection becomes more dominant there.

Figure 4 shows values of $\overline{\mathrm{Nu}}_{\text {tot }}$ for the illustrated cases in Figure 3. According to Figure 4(b), it is clear that the heat transfer rate increases with decreasing the nanoparticle diameter and the highest values of the heat transfer rate occur at $25 \mathrm{~nm}$ diameter. It is also noticed that by increasing volume fraction of nanoparticles and decreasing Ri the heat transfer rate increases. At $\mathrm{Ri}>10$ the effects of natural convection are dominant so there is an optimum volume fraction of nanoparticles which maximize the heat transfer which is about $3 \%$ in the most cases.

Figure 4(a) shows the effects of using different types of nanofluids on $\overline{\mathrm{Nu}}_{\text {tot }}$. In all Richardson numbers nonsignificant variation on the heat transfer rate is observed when different kinds of nanofluids are used. It can be seen that among all nanoparticles those nanoparticles with higher thermal conductivity (such as $\mathrm{Cu}$ ) produce slightly higher rate of heat transfer compared to nanoparticles with lower thermal conductivity (such as $\mathrm{TiO}_{2}$ ). It is clear from Figure 4 that, at all $\mathrm{Ri}$, if the direction of the two moving walls is same, the heat transfer rate increases. From Cases 2 to 1 , the achieved reduction of $\overline{\mathrm{Nu}}_{\text {tot }}$ is approximately $4.67 \%, 0.28 \%$, $0.09 \%$, and $0.44 \%$, at $\mathrm{Ri}=0.1,1,10$, and 100 , respectively. Consequently, the heat transfer rate variation between these two cases is negligible.

\section{Conclusion}

This study investigates mixed convection of the nanofluid in two-sided lid-driven square cavity with a pair of triangular heating cylinders. The effects of various design parameters such as Richardson number $(0.1 \leq \mathrm{Ri} \leq 100)$, nanoparticle volume fraction $(0 \leq \varphi \leq 0.05)$, and size $\left(25 \mathrm{~nm} \leq d_{p} \leq\right.$ $145 \mathrm{~nm})$ and type $\left(\mathrm{Cu}, \mathrm{Al}_{2} \mathrm{O}_{3}, \mathrm{TiO}_{2}\right)$ of nanoparticles on the 
$\mathrm{Ri}$
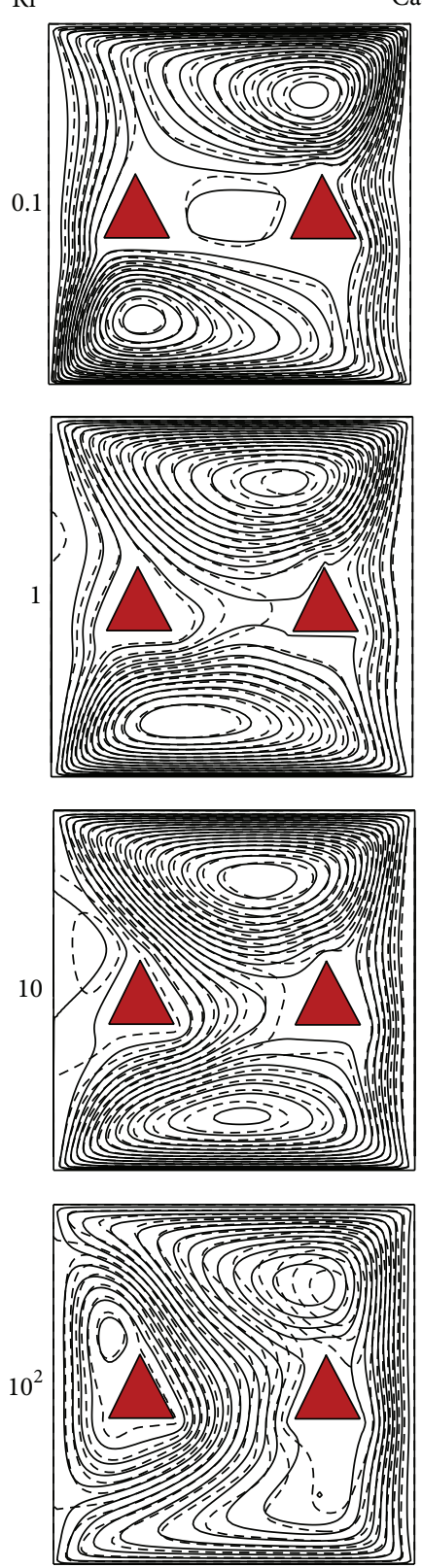

(a) Streamlines
Case 1
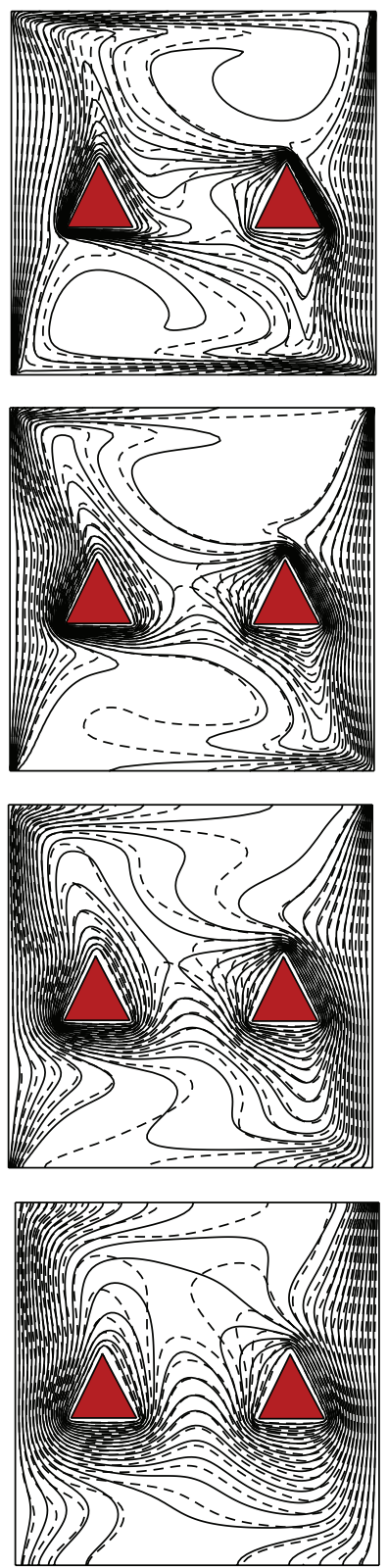

(b) Isotherms

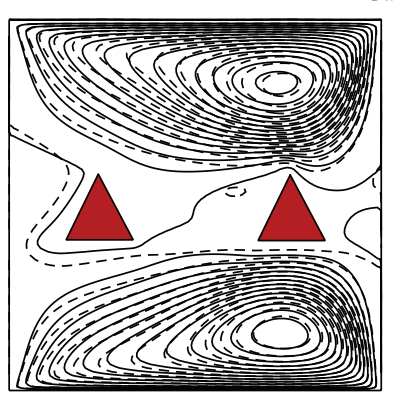

Case 2
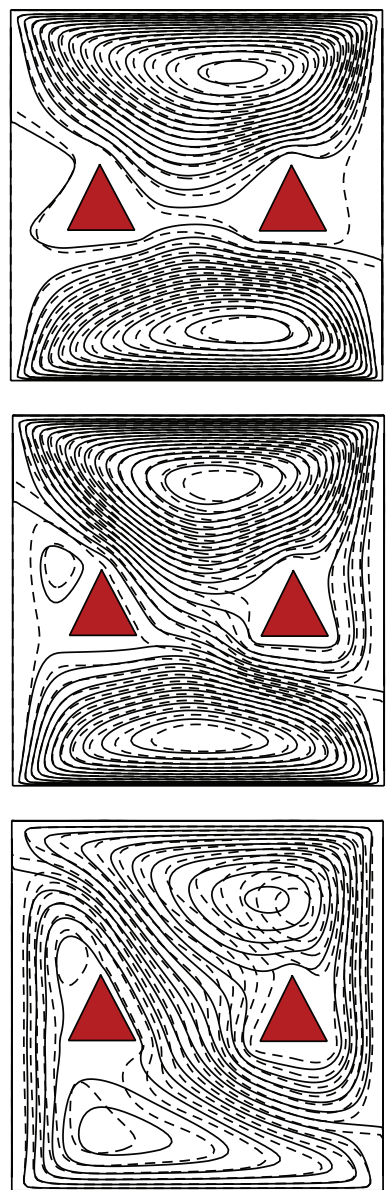

(a) Streamlines
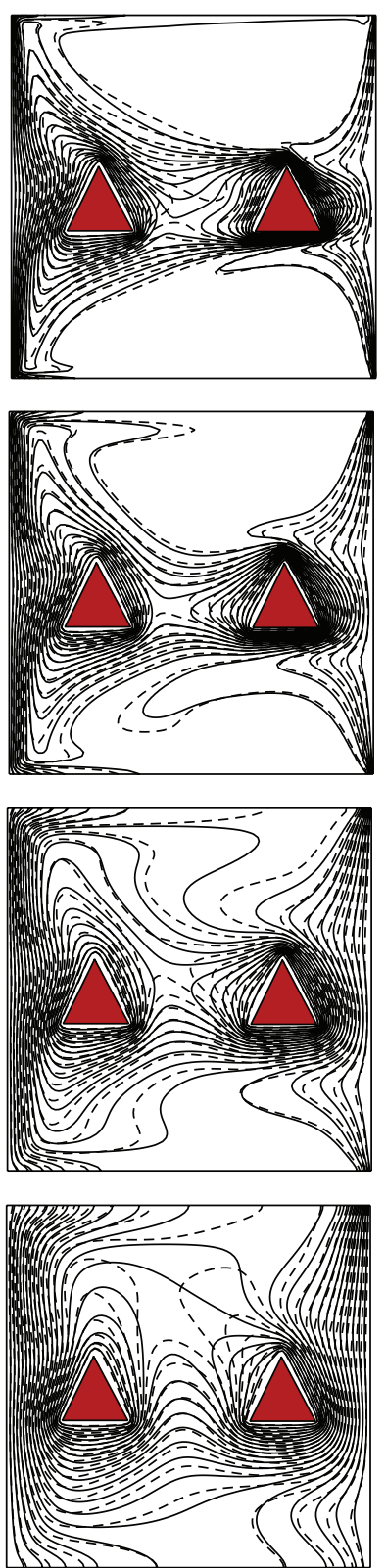

(b) Isotherms

FIGURE 3: (a) Streamlines and (b) isotherms inside the cavity with a pair of triangular heat sources $(W=w / H=0.2)$ filled with Cu-water pure fluid (dashed line) and $\mathrm{Cu}$-water nanofluid (solid line) with $\varphi=5 \%$ and at different Ris for Cases 1 and $2 . \mathrm{Gr}=10^{4}$.

heat transfer rate are investigated. According to the presented results, the following conclusions are drawn:

(i) By increasing volume fraction of nanoparticles $(\varphi)$ and increasing Richardson number ( $\mathrm{Ri})$, the heat transfer rate increases.

(ii) The heat transfer rate increases with decreasing the nanoparticle diameter and the highest values of the heat transfer rate occur at $25 \mathrm{~nm}$ diameter.

(iii) In all Richardson numbers nonsignificant variation on the heat transfer rate is observed when different kinds of nanofluids are used. It can be seen that among all nanoparticles those nanoparticles with higher thermal conductivity (such as $\mathrm{Cu}$ ) produce slightly higher rate of heat transfer compared to nanoparticles with lower thermal conductivity (such as $\mathrm{TiO}_{2}$ ).

(iv) At Ri > 10 the effects of natural convection are dominant so there is an optimum volume fraction of nanoparticles which maximize the heat transfer which is about $3 \%$ in the most cases.

(v) The effect of changing direction of the two horizontal moving walls on the heat transfer rate $\left(\overline{\mathrm{Nu}}_{\mathrm{tot}}\right)$ is negligible. 

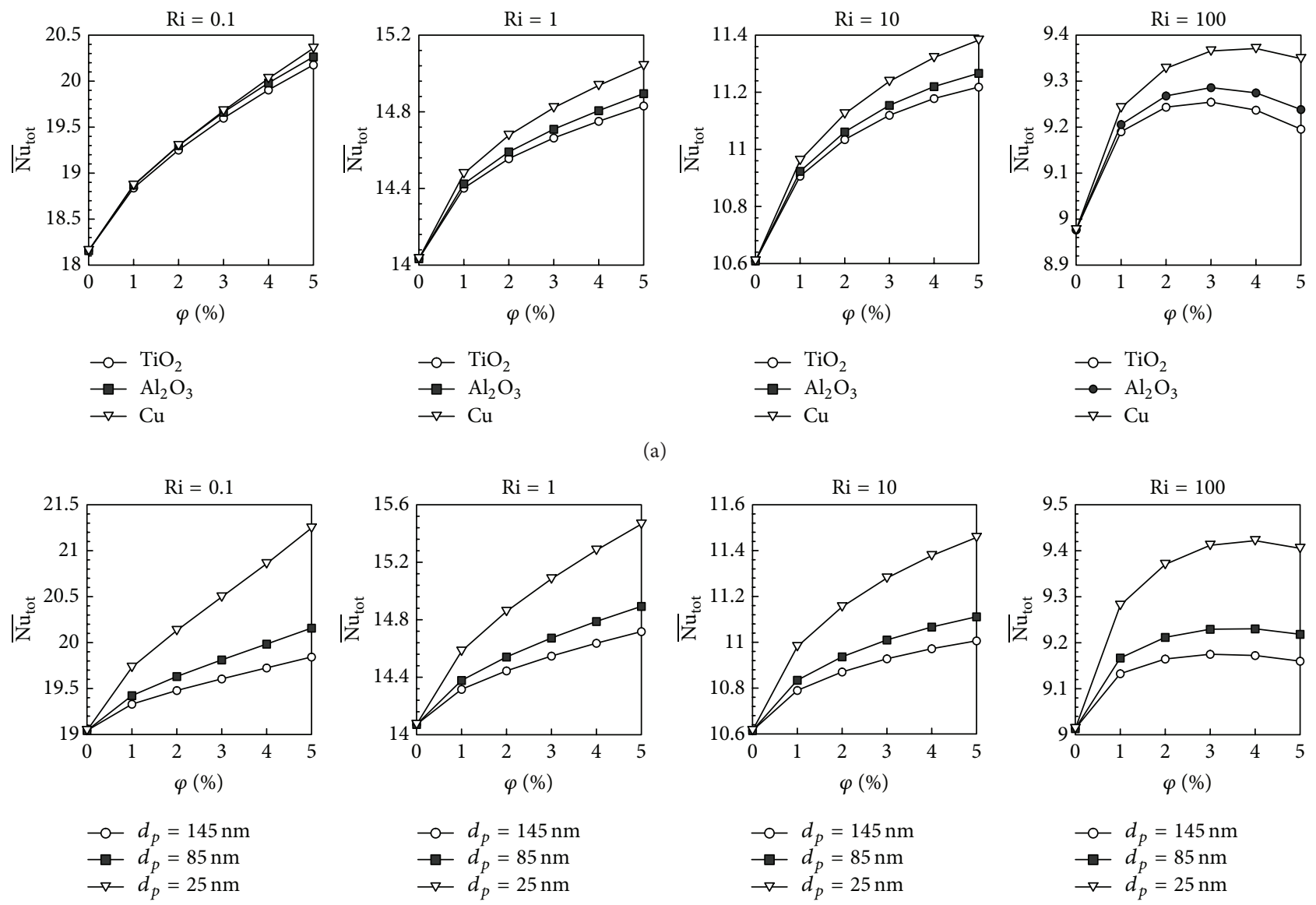

(b)

Figure 4: Variations $\overline{\mathrm{Nu}}_{\text {tot }}$ with respect to the volume fraction of the nanoparticles at different Richardson numbers, diameters, and type of nanoparticles for (a) Case 1 and (b) Case 2. Gr $=10^{4}$.

\section{Nomenclature}

$C_{p}: \quad$ Specific heat, $\mathrm{Jkg}^{-1} \mathrm{~K}^{-1}$

$d_{p}$ : Diameter of the nanoparticle, $\mathrm{m}$

$d_{f}$ : Diameter of the base fluid molecule, $\mathrm{m}$

g: Gravitational acceleration, $\mathrm{ms}^{-2}$

Gr: $\quad$ Grashof number $\left(=g \beta \Delta T H^{3} / \nu^{2}\right)$

$H$ : Enclosure height, $\mathrm{m}$

$k$ : Thermal conductivity, $\mathrm{Wm}^{-1} \mathrm{~K}^{-1}$

$k_{b}$ : Boltzmann's constant $=1.38066 \times 10^{-23}$

$\overline{\mathrm{Nu}}_{\text {tot }}$ : Heat transfer of cavity's wall

$p$ : Dimensional pressure, $\mathrm{Nm}^{-2}$

$P$ : Dimensionless pressure

Pr: $\quad$ Prandtl number $\left(=\nu_{f} / \alpha_{f}\right)$

Ra: $\quad$ Rayleigh number $\left(=g \beta_{f}\left(T_{h}-T_{c}\right) H^{3} / \alpha_{f} \nu_{f}\right)$

$\mathrm{Re}_{B}$ : Brownian-motion Reynolds number

Re: $\quad$ Reynolds number $\left(=U_{0} H / \nu\right)$

Ri: $\quad$ Richardson number $\left(=\mathrm{Gr} / \mathrm{Re}^{2}\right)$

T: $\quad$ Temperature, $\mathrm{K}$

$t$ : Time, s

$\tau: \quad$ Dimensionless time $\left(t / H / U_{\text {ref }}\right)$

$u, v: \quad$ Velocity components, $\mathrm{ms}^{-1}$
$u_{B}$ : Brownian velocity of the nanoparticle, $\mathrm{ms}^{-1}$

$U, V:$ Dimensionless velocity components

$w$ : Dimensional width of the triangular heater

$W$ : Dimensionless width of the triangular heater

$x, y$ : Cartesian coordinates, $\mathrm{m}$

$X, Y$ : Dimensionless Cartesian coordinates $(x$, y) $/ H$.

\section{Greek Symbols}

$\alpha$ : Thermal diffusivity, $\mathrm{m}^{2} \mathrm{~s}^{-1}$

$\beta$ : Thermal expansion coefficient, $\mathrm{K}^{-1}$

$\theta$ : Dimensionless temperature

$\mu$ : Dynamic viscosity, $\mathrm{kgm}^{-1} \mathrm{~s}^{-1}$

$\nu$ : Kinematic viscosity, $\mathrm{m}^{2} \mathrm{~s}^{-1}$

$\rho$ : Density, $\mathrm{kgm}^{-3}$

$\varphi$ : Volume fraction of the nanoparticles

$\psi$ : Stream function $\left(=-\int_{Y_{o}}^{Y} U \partial Y+\psi\left(X, Y_{0}\right)\right)$. 


\author{
Subscripts \\ c: Cold \\ $f$ : Fluid \\ $h$ : Hot \\ nf: Nanofluid \\ p: Solid nanoparticles.
}

\section{Conflict of Interests}

The authors declare that there is no conflict of interests regarding the publication of this paper.

\section{References}

[1] H. F. Oztop, I. Dagtekin, and A. Bahloul, "Comparison of position of a heated thin plate located in a cavity for natural convection," International Communications in Heat and Mass Transfer, vol. 31, no. 1, pp. 121-132, 2004.

[2] K. Khanafer and S. M. Aithal, "Laminar mixed convection flow and heat transfer characteristics in a lid driven cavity with a circular cylinder," International Journal of Heat and Mass Transfer, vol. 66, pp. 200-209, 2013.

[3] A. W. Islam, M. A. R. Sharif, and E. S. Carlson, "Mixed convection in a lid driven square cavity with an isothermally heated square blockage inside," International Journal of Heat and Mass Transfer, vol. 55, no. 19-20, pp. 5244-5255, 2012.

[4] M. Kalteh, K. Javaherdeh, and T. Azarbarzin, "Numerical solution of nanofluid mixed convection heat transfer in a liddriven square cavity with a triangular heat source," Powder Technology, vol. 253, pp. 780-788, 2014.

[5] M. El Abdallaoui, M. Hasnaoui, and A. Amahmid, "Numerical simulation of natural convection between a decentered triangular heating cylinder and a square outer cylinder filled with a pure fluid or a nanofluid using the lattice Boltzmann method," Powder Technology, vol. 27, pp. 193-205, 2015.

[6] M. El Abdallaoui, M. Hasnaoui, and A. Amahmid, "Latticeboltzmann modeling of natural convection between a square outer cylinder and an inner isosceles triangular heating body," Numerical Heat Transfer, Part A: Applications, vol. 66, no. 9, pp. 1076-1096, 2014.

[7] H. F. Oztop, Z. Zhao, and B. Yu, "Fluid flow due to combined convection in lid-driven enclosure having a circular body," International Journal of Heat and Fluid Flow, vol. 30, no. 5, pp. 886-901, 2009.

[8] M. Corcione, "Empirical correlating equations for predicting the effective thermal conductivity and dynamic viscosity of nanofluids," Energy Conversion and Management, vol. 52, no. 1, pp. 789-793, 2011.

[9] M. Corcione, "Heat transfer features of buoyancy-driven nanofluids inside rectangular enclosures differentially heated at the sidewalls," International Journal of Thermal Sciences, vol. 49, no. 9, pp. 1536-1546, 2010.

[10] Z. Haddad, H. F. Oztop, E. Abu-Nada, and A. Mataoui, "A review on natural convective heat transfer of nanofluids," Renewable and Sustainable Energy Reviews, vol. 16, no. 7, pp. 5363-5378, 2012.

[11] S. V. Patankar, Numerical Heat Transfer and Fluid Flow, McGraw-Hill, Washington, DC, USA, 1980.

[12] D. B. Spalding, "A novel finite difference formulation for differential expressions involving both first and second derivatives,"
International Journal for Numerical Methods in Engineering, vol. 4, no. 4, pp. 551-559, 1972.

[13] R. Iwatsu, J. M. Hyun, and K. Kuwahara, "Mixed convection in a driven cavity with a stable vertical temperature gradient," International Journal of Heat and Mass Transfer, vol. 36, no. 6, pp. 1601-1608, 1993.

[14] F. P. Incropera and D. P. DeWitt, Introduction to Heat Transfer, John Wiley \& Sons, New York, NY, USA, 2002. 


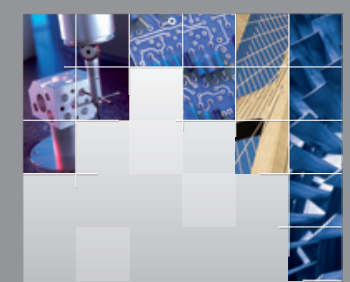

\section{Enfincering}
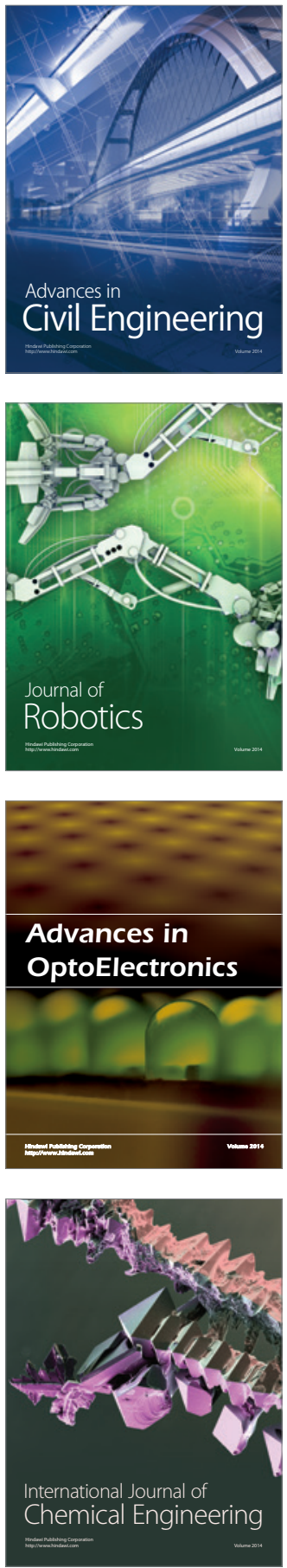

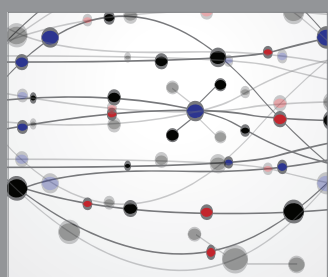

The Scientific World Journal

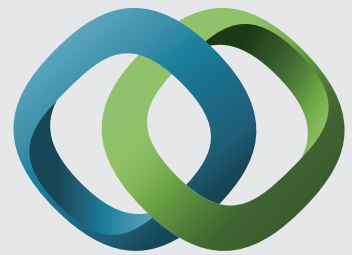

\section{Hindawi}

Submit your manuscripts at

http://www.hindawi.com
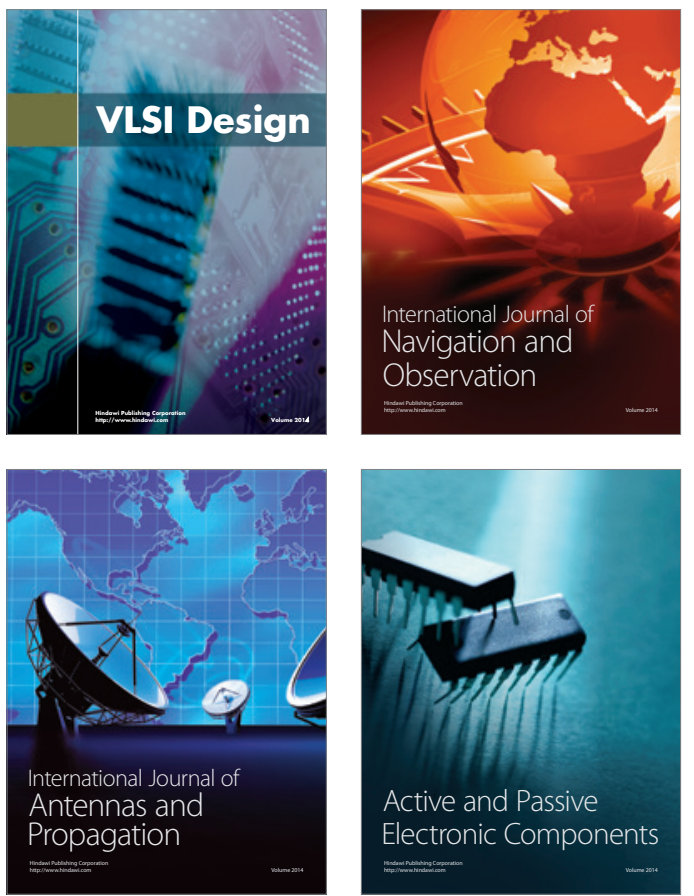
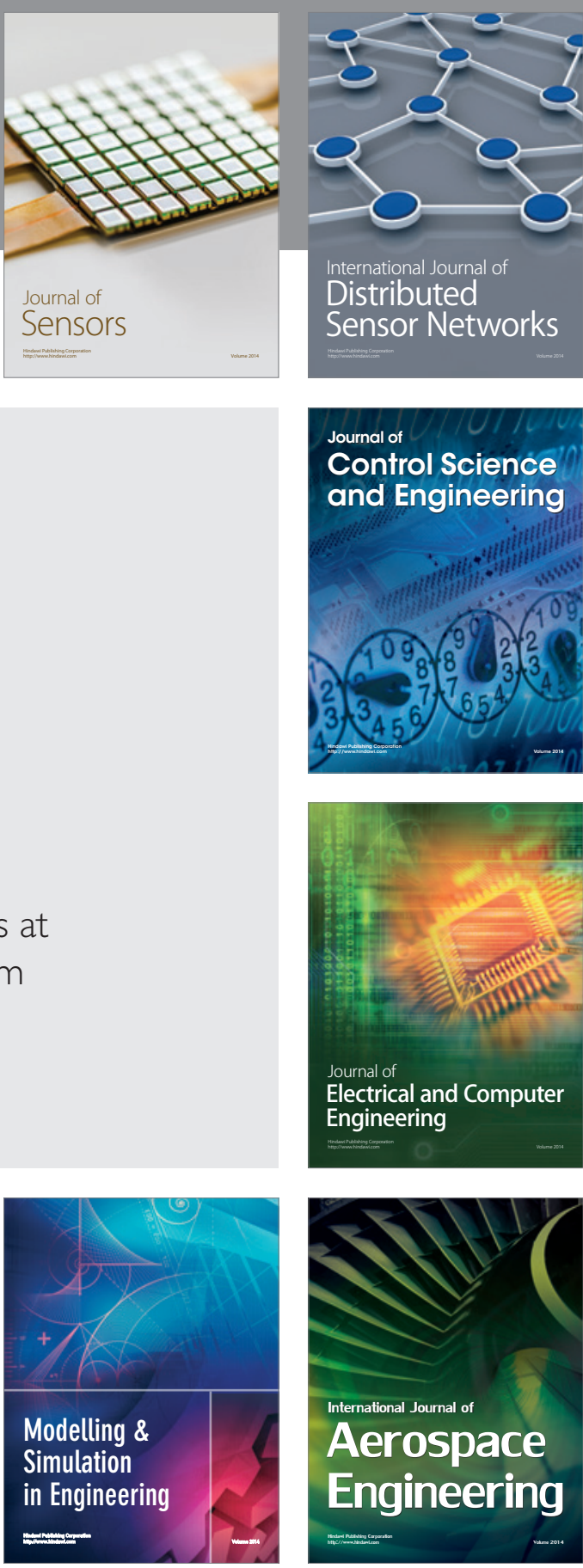

International Journal of

Distributed

Sensor Networks

Journal of

Control Science

and Engineering
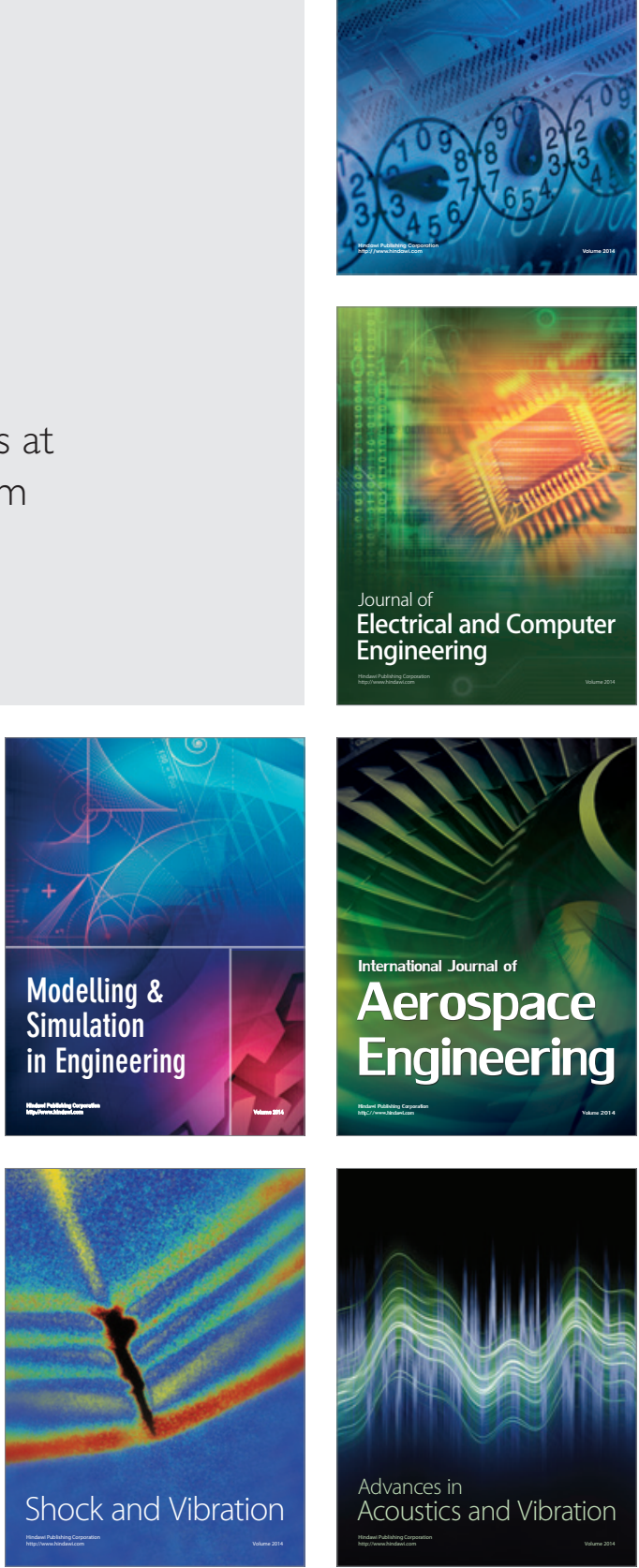